\title{
FREE RADICAL GRAFT MODIFICATION OF POLYETHYLENE WITH METHACRYLIC ACID AND STYRENE MONOMER
}

\author{
M. Shahzad Kamal*, G. M. Mamoor, Nida Qamar, Muhammad Farooq, S. Muhammad \\ Polymer and Process Engineering Department, University of Engineering E Technology Lahore, Pakistan
}

Received 10 April 2011; accepted 9 August 2011

\begin{abstract}
Polar groups can be introduced on polyethylene by using polar monomer like acryl amide, acrylic acid and methacrylic acid. In present work binary monomer mixture of methacrylic acid and styrene were grafted onto low density polyethylene in the presence of dicumyl peroxide (DCP) as an initiator. Grafted polyethylene was characterized by using differential scanning calorimetry (DSC), melt flow Indexer (MFI) and Fourier transform infrared spectroscope (FTIR). Low value of MFI as compared to virgin polyethylene, presence of carbonyl peaks in the spectrum of grafted polyethylene and higher endothermic peaks in thermogram of grafted polyethylene confirmed grafting of methacrylic acid and styrene onto polyethylene. MFI values at different loads were used for further rheological study of virgin and grafted polyethylene.
\end{abstract}

Keywords: Degree of grafting, Methacrylic acid, Free radical, Vinyl monomer, DCP

DOI:10.3329/cerb.v15i1.7419

\section{Introduction}

Polyolefins are most extensively used low cost thermoplastics having excellent bulk properties and are used in a wide range of applications [1-5]. Despite of their versatility, absence of special surface properties such as printability, hydrophilicity, poor dispersibility with inorganic fillers, roughness, wettability, lubricity and selective permeability are absent in these valuable commodity thermoplastics due to their inert and non polar nature $[2,3]$. These drawbacks limit the use of these materials for special applications like printed circuit, diffusion barrier coating, decorative coating, membrane for separation, conducting polymers and many more $[5,6]$. Therefore surface modification of polyolefins, have been comprehensively studied for last fifty years [7].

Large number of techniques have been proposed for surface modification of polyolefins like blending, grafting, and curing. Among the existing methods of modification of polyolefins, grafting is an attractive method to impart a variety of functional groups on to a polyolefin surface $[6,8,9]$. Extensive work has been done on techniques of graft co-polymerization of different monomers on polyolefin backbone. These techniques include chemical, radiation, photochemical, plasma-induced and enzymatic grafting [6]. In present work free radical grafting of styrene and meth acrylic acid in presence of dicumyl peroxide as an initiator was done onto polyethylene. Number of researchers [10-13] have reported chemical grafting of

*Corresponding author Email: shaizmalik41@yahoo.com single monomer on polyethylene but little work has been done on binary monomer mixture grafting particularly by using mixture of styrene and meth acrylic acid.

\section{Experimental}

\subsection{Materials}

Basic materials used in these experiments were low density polyethylene (LDPE), methacrylic acid (MAA), styrene, and dicumyl peroxide (DCP). LDPE used as a base polymer was imported from Qatar Petrochemical Company Ltd. The LDPE has MFI of 0.7 (g/10min) and specific gravity of 0.94-0.97. Methacrylic Acid used as monomer having 98\% purity was obtained from Acros Organic. Co monomer Styrene of $99 \%$ purity was supplied by Alfa Aesar. Dicumyl peroxide (98\%) used as an initiator was taken from Sigma Aldrich.

\subsection{Synthesis of grafted polyethylene}

Weighed amount of PE, styrene, water and methacrylic acid were placed in a round bottom flask. The system temperature was increased up to $93^{\circ} \mathrm{C}$. As the desired temperature was attained DCP was added to the mixture and thorough agitation was carried out. The mixture was kept at the reaction temperature for a period of 3 hours. After completion of reaction, product was washed with $0.1 \mathrm{~N} \mathrm{NaOH}$, dried then washed with water followed by washing with $0.1 \mathrm{~N} \mathrm{HCl}$, dried and again washed with water. The sample is then finally dried in an oven and further analysis was carried out. Composition of mixture is shown in Table 1.

(C)Bangladesh Uni. of Engg. E Tech. 
Table 1: Composition of the mixture

\begin{tabular}{lr}
\hline Materials & Amounts \\
\hline Methacrylic acid & $15 \mathrm{ml}$ \\
Styrene & $1.66 \mathrm{ml}$ \\
DCP & $0.75 \mathrm{~g}$ \\
Water & $35 \mathrm{ml}$ \\
PE & $50 \mathrm{~g}$ \\
\hline
\end{tabular}

\subsection{FTIR Spectroscopy}

The FTIR spectra were recorded on JASCO FT/IR4100 infrared spectrometer. Potassium bromide (KBr) was used as an inert background material to get the spectrums of both pure and grafted samples of polyethylene. The analysis was done in the region of 400 to $4000 \mathrm{~cm}^{-1}$.

\subsection{Differential Scanning Calorimetry (DSC)}

DSC curves were obtained at heating rate of $10^{\circ} \mathrm{C}$ $\min ^{-1}$ in Shimadzu DSC 60-A apparatus. Both pure and grafted samples were analyzed against aluminum as reference material. The temperature range for the experiment was selected to be $180^{\circ} \mathrm{C}$.

\subsection{Rheological Testing}

MFI of the grafted and pure sample was determined by using MFI apparatus (KARG Industrietechnik) according to ASTM D 1238-04 under load of 2.16 to $21.6 \mathrm{~kg}$ with die diameter of $2.095 \mathrm{~mm}$ and at $190^{\circ} \mathrm{C}$ temperature.

\section{Results and Discussion}

\subsection{FTIR spectroscopy}

An important study regarding the proof of the efficient grafting of polyethylene is the infrared spectroscopy. FTIR spectrum of virgin polyethylene (Figure 1) shows characteristic peaks at 721 and 1642 $\mathrm{cm}^{-1}$ which indicates methylene group rocking and session vibrations respectively while symmetric and asymmetric methylene group stretching was observed at 2844 and $2918 \mathrm{~cm}^{-1}$ respectively. While in spectrum of grafted polyethylene (Figure 2) peak present at $1697 \mathrm{~cm}^{-1}$ is of considerable importance which confirms the presence of $\mathrm{COOH}$ group. This $\mathrm{COOH}$ group peak is due to presence of meth acrylic acid confirming grafting of meth acrylic acid on polyethylene.

\subsection{Differential Scanning Calorimetry (DSC)}

Figure 3 shows thermograms of ungrafted polyethylene and grafted polyethylene .From this figure melting peak of LDPE was found at $110.09^{\circ} \mathrm{C}$ while for grafted polyethylene melting point was observed at $111.15^{\circ} \mathrm{C}$ which is higher than that of the ungrafted polyethylene. This increase in melting point can be attributed to the presence of polar groups generated after

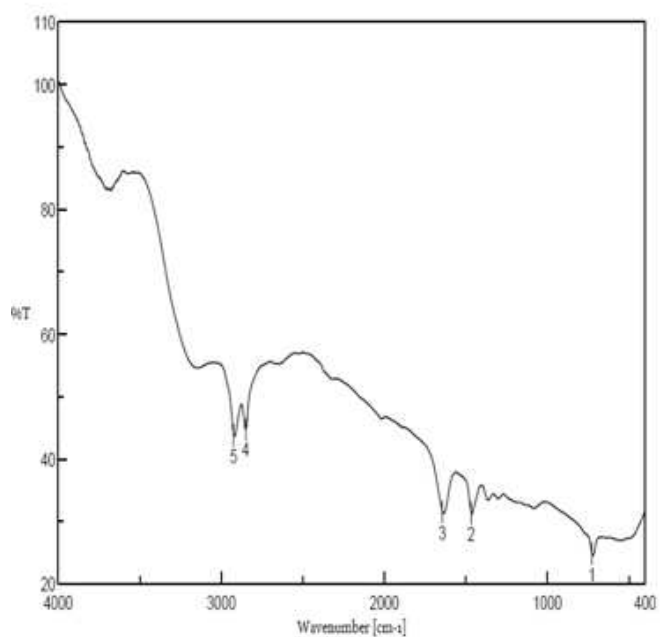

Figure 1: FTIR spectrum of polyethelene(ungrafted)

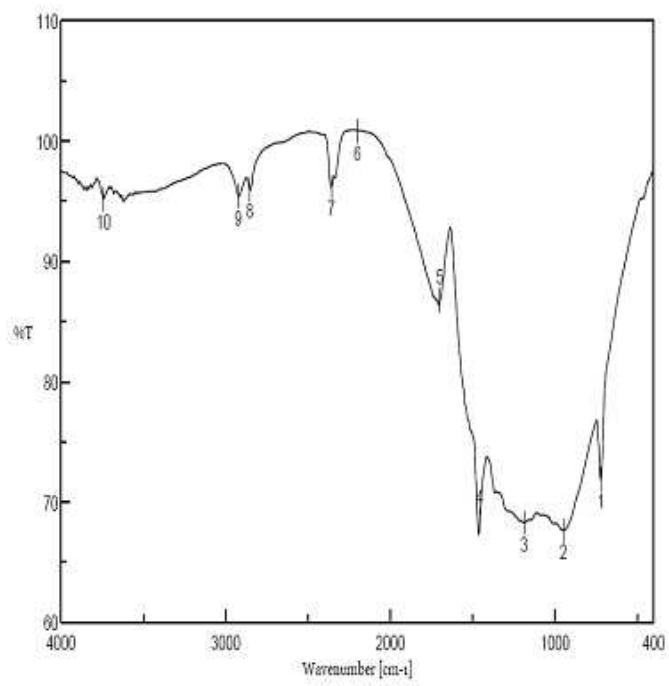

Figure 2: FTIR spectrum of grafted polyethylene

grafting. Another important point is that this change in melting point, however, is relatively low and can be ascribed to low level of grafting. On a molecular level, the grafted chains are relatively difficult to move, rotate, and oscillate as compared to ungrafted ones; thus elevating the melting point of the material. Another interesting point to be noted here is that the thermogram of grafted polyethylene prior to melting point peak is not as smoother as in case of ungrafted polyethylene. This is because the movement of grafted side chains starts before the movement of main chain. It means a little bit more amount of energy is absorbed for the movement of these grafted side chains prior to the movement of main chain. Conversely, we can infer two types of movements of the chains are to be occurred there: first one for the movement of small grafted side chains followed by the movement of main 


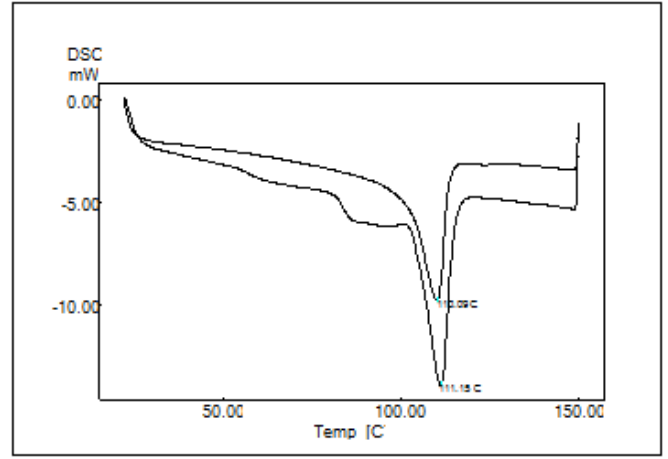

Figure 3: DSC curves of (upper) Virgin polyethylene and (lower) grafted polyethylene

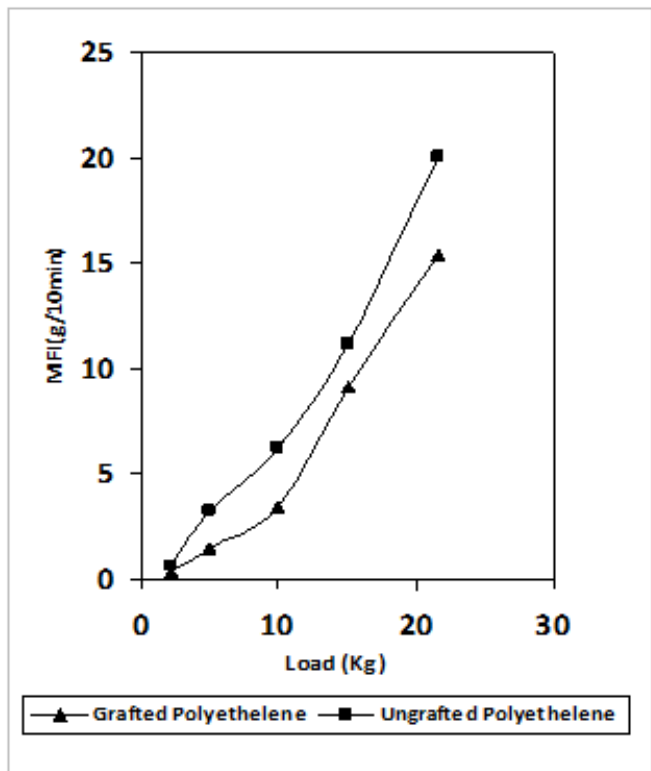

Figure 4: MFI (g/10minute) as a function of load $(\mathrm{kg})$

backbone chains. In the first case a small quantity of heat is absorbed to melt the smaller side chains, but a large quantity of heat is engaged in the second case.

\subsection{Rheological Properties}

Rheological measurements are very important for quality control of raw materials, optimization of manufacturing processes and forecasting the performance of a material [14]. Melt flow index (MFI) is a good indicator of flow properties of the material under consideration. Figure 4 shows MFI of both grafted and ungrafted polyethylene at different loads. It is evident from the Figure 4 that MFI of ungrafted polyethylene is more than grafted polyethylene at different loads.

This decrease in MFI after grafting is associated with the greater degree of branching of grafted polyethylene as compared to ungrafted polyethylene. Presence of branches diminishes the flow properties in polyethylene up to much extent. This drop off in MFI is also an indication of efficient grafting of MAA and styrene on polyethylene.

Shear stress and strain rate can be calculated by using Equations 1 and 2 respectively:

$$
\begin{gathered}
\tau=\frac{R_{N} F}{2 \pi R_{P}^{2} L_{N}} \\
\gamma=\frac{4 Q}{\pi R_{N}^{3}}
\end{gathered}
$$

where $R_{N}$ is radius of nozzle $(0.105 \mathrm{~cm}), L_{N}$ is nozzle length $(0.8 \mathrm{~cm}), R_{P}$ is piston radius $(0.4737 \mathrm{~cm}), Q$ is flow rate $\left(\mathrm{cm}^{3} / \mathrm{sec}\right)$ and $F$ is test load $(\mathrm{kg}) \times 9.087 \times$ 105 dyne. Stress strain curves obtained from MFI data are represented in Figure 5. By increasing the strain rate, stress for both the grafted as well as ungrafted polyethylene increases accordingly, the relative difference between stresses for grafted and ungrafted counterparts being small at zero strain rate. As strain rate exceeds zero, grafted polyethylene requires extra amount of stress than its ungrafted counterpart to make the same amount of deformation. In case of grafted polyethylene, resistance offered by grafted chains to flow and deform is the major reason of higher stress value. It is evident from Figure 5 that the behaviour of both grafted and virgin polyethylene is pseudoplastic with yield stress. For the case of flow of polymer through circular die, a correction is normally applied to take into account pseudoplastic nature of the melt which implies that the assumed parabolic profile in the die actually illustrates more plug flow behavior [15]. This correction is generally expressed as:

$$
\Upsilon_{\text {true }}=\frac{4 Q}{\pi R^{3}} \frac{(3 n+1)}{4 n}
$$

Viscosity can be calculated from the following equation:

$$
\eta_{a}=m \gamma^{\mathrm{n}-1}
$$

where, $\eta_{a}$ is the viscosity.

From Figure 6 it is apparent that viscosity decreases with the increase in strain rate for both the virgin as well as the grafted polyethylene grades. The viscosity of grafted polyethylene is approximately one and a half times the viscosity of virgin counterpart at zero strain rate. Although the magnitude of viscosity for both the grades varies with strain rate, relative difference between the viscosity of grafted and pure polyethylene counterparts remains almost same for the entire range of strain rate. Higher viscosity of grafted polyethylene than virgin polyethylene is attributed to the presence of branches in grafted polyethylene which offer more hindrance to chain alignment in flow direction on applying stress as compared to the more flexible straight chains in ungrafted polyethylene. Grafted chains make some sort of physical entanglements among themselves which stand firm 


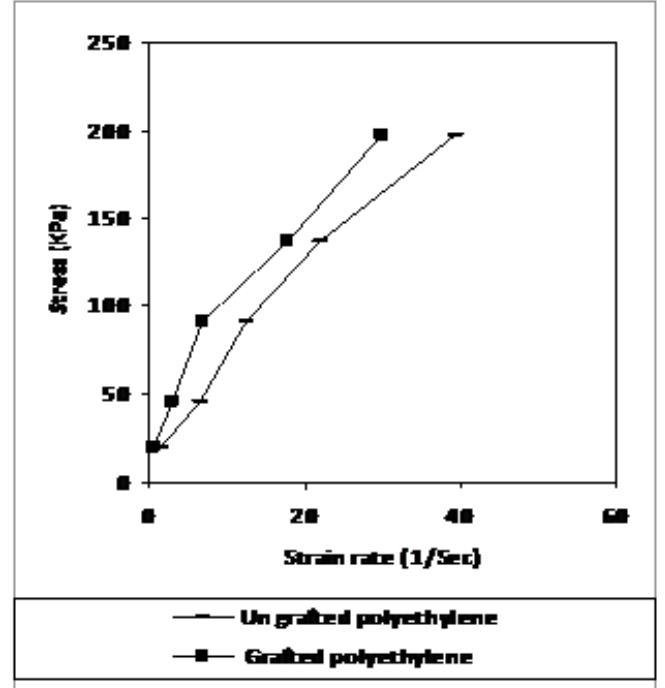

Figure 5: Strain rate $(1 / \mathrm{Sec})$ as a function of stress $(\mathrm{KPa})$

against applied stress, thus increasing melt viscosity. This decrease in viscosity with increasing strain rate reveals rheopectic behavior for both the grades, the grafted polyethylene demonstrating more rheopecticity (fluid consistency index $=0.6$ ) than the virgin polyethylene counterpart (fluid consistency index = $0.7)$.

\section{Conclusion}

From above discussion it can be inferred that reasonable grafting can be achieved by using binary monomer mixture of styrene and methacrylic acid. FTIR analysis shows presence of $\mathrm{COOH}$ group in grafted polyethylene confirming grafting of methacrylic acid and styrene. Rheological properties have been changed due to grafting which is indicated by lower value of MFI of grafted polyethylene as compared to virgin polyethylene. For same strain rate, higher stress is required for grafted polyethylene as compared to virgin polyethylene due to resistance in flow offered by branched chains. Viscosity also increases by introduction of branches. From DSC analysis it is concluded that melting point of polyethylene increases after grafting.

\section{Acknowledgement}

The authors are thankful to the Department of Polymer And Process Engineering, University Of Engineering and Technology Lahore Pakistan for the Laboratory support.

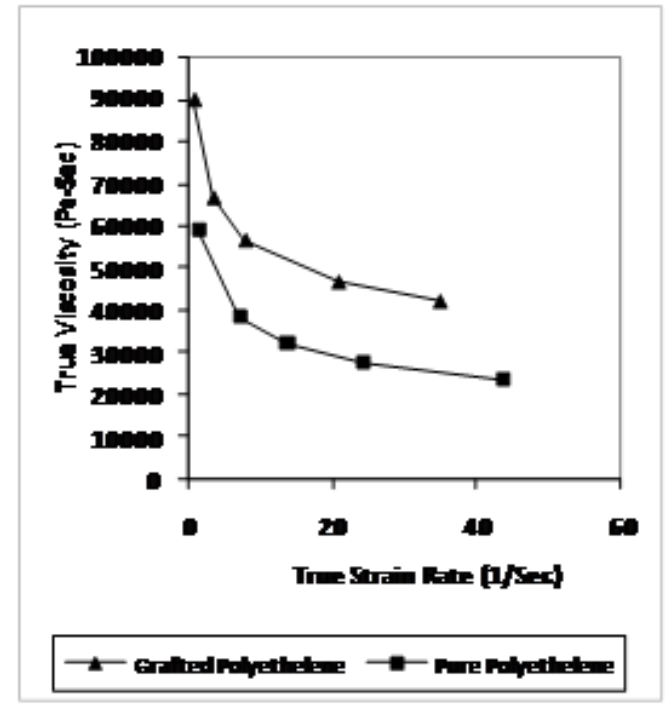

Figure 6: True strain rate $(1 / \mathrm{sec})$ as a function of true viscosity $(\mathrm{Pa}-$ $\sec )$

\section{References}

[1] Burnett DJ, Thielmann F and Ryntz RA, Correlating thermodynamic and mechanical adhesion phenomena for thermoplastic polyolefins, Journal of Coatings Technology and Research, 2007. 4(2):pp. 211-215

[2] Desai SM and Singh RP, Surface Modification of Polyethylene, in Long Term Properties of Polyolefins, ed. Albertsson AC, Advances in Polymer Science, Vol. 169, Springer Berlin / Heidelberg, 2004. pp. 231-294

[3] Singh SK, Tambe SP, Samui AB, Raja VS and Kumar D, Maleic acid grafted low density polyethylene for thermally sprayable anticorrosive coatings, Progress in organic coatings, 2006. 55(1):pp. 20-26

[4] de O Meraes G, Geraldes AN, Zen HA, Parra DF and BLugao A, Preliminary study of styrene grafting on Polyethylene films, in International Nuclear Atlantic Conference, Santos, SP, Brazil, 2007

[5] Shojaei A, Fathi R and Sheikh N, Adhesion modification of polyethylenes for metallization using radiation-induced grafting of vinyl monomers, Surface and Coatings Technology, 2007. 201(16-17):pp. 7519-7529

[6] Bhattacharya A and Misra BN, Grafting: a versatile means to modify polymers:: Techniques, factors and applications, Progress in polymer science, 2004. 29(8):pp. 767-814

[7] Ranogajec F, Effect of solvent on radiation grafting and crosslinking of polyethylene, Radiation Physics and Chemistry, 2007. 76(8-9):pp. 1381-1384

[8] Uyama Y, Kato K and Ikada Y, Surface Modification of Polymers by Grafting, in Grafting/Characterization Techniques/Kinetic Modeling, eds. Galina H, Ikada Y, Kato K, Kitamaru R, Lechowicz J, Uyama $\mathrm{Y}$ and Wu $\mathrm{C}$, Advances in Polymer Science, Vol. 137, Springer Berlin / Heidelberg, 1998. pp. 1-39

[9] Teke AB and Baysal SH, Immobilization of urease using glycidyl methacrylate grafted nylon-6-membranes, Process Biochemistry, 2007. 42(3):pp. 439-443

[10] Yamada K, Kimura T, Tsutaya H and Hirata M, Hydrophilic and adhesive properties of methacrylic acid-grafted polyethylene plates, Journal of applied polymer science, 1992. 44(6):pp. 993-1001

[11] Hsiue GH and Huang WK, Preirradiation grafting of acrylic and methacrylic acid onto polyethylene films: Preparation 
and properties, Journal of applied polymer science, 1985. 30(3):pp. 1023-1033

[12] Lawler J and Charlesby A, Grafting of acrylic acid onto polyethylene using radiation as initiator, Radiation Physics and Chemistry (1977), 1980. 15(5):pp. 595-602

[13] Tretinnikov O, Analysis of photoinduced graft polymerization of acrylic acid onto polyethylene films by attenuated total reflectance IR spectroscopy, Journal of Applied Spectroscopy, 2009. 76:pp. 898-901. doi:10.1007/s10812-010-9268-x

[14] Shenoy AV and Saini DR, Thermoplastic melt rheology and processing, CRC Press, 1996

[15] Cogswell FN, Polymer melt rheology: a guide for industrial practice, Woodhead Publishing, 1981 